\title{
EDITORIAL
}

\section{Transitions and Transformations}

The year 2011 brings a number of changes to Neurotherapeutics. When initiated in January 2004, the journal was self-published by the American Society for Experimental NeuroTherapeutics (ASENT). For the past five years, Elsevier has been our publisher. During that time, the journal's visibility has increased markedly, with monthly downloads presently exceeding 12,000 per month. In June 2010, Neurotherapeutics received a highly competitive initial impact factor of 5.38, placing it among the top $10-15 \%$ of journals in both the basic and clinical neuroscience areas.

Although we had an excellent working relationship with Elsevier, our contract was up for renegotiation, and the leadership of ASENT and its Publication Oversight Committee elected to solicit proposals from multiple publishers for the new contract term. The ultimate decision to choose Springer as our new publisher reflected, in part, its willingness to provide increased editorial manager support and its commitment to facilitate the addition of primary research articles with early online publication.

Neurotherapeutics was initially developed to provide a unique platform for publishing comprehensive, topicspecific, critical reviews related to translational neuroscience. However, it was always planned that after achieving sufficient visibility and a competitive impact factor, the journal would also begin to include original translational research articles of exceptional quality. Publication of such research papers will begin this year. These submissions will be quickly reviewed, with accepted manuscripts rapidly published online, as well as in the following quarterly print edition. For investigators doing translational neuroscience, the choice of journal in which to publish often proves problematic. It is hoped that Neurotherapeutics will help fulfill this need by providing a high visibility, high impact platform.

I wish to express my appreciation to: our outstanding Associate Editors Rohit Bakshi and Michael Rogawski; Linda Powell, who has provided exceptional editorial support since the journal's inception; our excellent and recently expanded editorial board; Morna Conway, who has expertly taken us through two publisher transitions; and our prior and present publishers Herb Niemirow at Elsevier and Matt Giampoala at Springer, whose professionalism and attention to detail have facilitated the journal's transition.

Alan I. Faden, M.D. Editor-in-Chief 